\title{
First-principles study of Dirac and Dirac-like cones in phononic and photonic crystals
}

\author{
Jun Mei, ${ }^{1,3, *}$ Ying Wu, ${ }^{2,3, \dagger}$ C. T. Chan, ${ }^{3, \ddagger}$ and Zhao-Qing Zhang ${ }^{3, \S}$ \\ ${ }^{1}$ Department of Physics, South China University of Technology, Guangzhou 510641, China \\ ${ }^{2}$ Division of Mathematical and Computer Sciences and Engineering, King Abdullah University of Science and Technology (KAUST), \\ Thuwal 23955-6900, Saudi Arabia \\ ${ }^{3}$ Department of Physics and William Mong Institute of Nano Science and Technology, Hong Kong University of Science and Technology, \\ Clear Water Bay, Hong Kong \\ (Received 7 February 2012; published 24 July 2012)
}

\begin{abstract}
By using the $\vec{k} \cdot \vec{p}$ method, we propose a first-principles theory to study the linear dispersions in phononic and photonic crystals. The theory reveals that only those linear dispersions created by doubly degenerate states can be described by a reduced Hamiltonian that can be mapped into the Dirac Hamiltonian and possess a Berry phase of $-\pi$. Linear dispersions created by triply degenerate states cannot be mapped into the Dirac Hamiltonian and carry no Berry phase, and, therefore should be called Dirac-like cones. Our theory is capable of predicting accurately the linear slopes of Dirac and Dirac-like cones at various symmetry points in a Brillouin zone, independent of frequency and lattice structure.
\end{abstract}

DOI: 10.1103/PhysRevB.86.035141

PACS number(s): 43.35.+d, 03.65.Ta, 42.70.Qs, 63.20.-e

\section{INTRODUCTION}

Graphene has attracted much attention in the past several years partly due to its intriguing transport properties, such as Klein tunneling, Zitterbewegung, antilocalization, abnormal quantum Hall effect, etc., arising from its unique band structures where the $E-k$ relation is linear at the six corners of the hexagonal Brillouin zone. ${ }^{1}$ The existence of Dirac cones in graphene can be well understood by using a tight-binding model for carbon atoms in a honeycomb lattice. ${ }^{1}$ Recently, Dirac cones in photonic and phononic crystals have also been found at the corners of the Brillouin zones of triangular and honeycomb lattices where two bands meet, ${ }^{2-9}$ leading to the observation of many novel wave transport properties, such as classical analogs of Zitterbewegung and pseudodiffusion. It was reported that linear dispersions can also occur at the Brillouin zone center of a square lattice photonic crystal, induced by simultaneous zero permittivity $\left(\varepsilon_{\text {eff }}=0\right)$ and permeability $\left(\mu_{\mathrm{eff}}=0\right)$, and the linear dispersions could be understood from an effective medium perspective. ${ }^{10}$ Different from the Dirac cone near the $K$ point of a triangular/honeycomb lattice, which is a result of double degeneracy, the existence of the linear dispersions near the $\Gamma$ point of a square lattice is a result of triple degeneracy, i.e., accidental degeneracy of a doubly degenerate mode and a single mode. We call this type of linear dispersion Dirac-like cones. Very recently, the Dirac-like cone has also been found in elastic/acoustic waves ${ }^{11}$ and in the simple cubic lattice. ${ }^{12}$ In the past few years, various transport properties of zero-refractive-index metamaterials have been studied near the Dirac-like point and the Dirac equation was widely assumed ${ }^{13}$ for those studies. The Dirac cones have also been found in many other photonic or plasmonic crystals in various dimensions. ${ }^{14-20}$

The existence of linear dispersions at some symmetry points of a Brillouin zone is much more common in classical waves than in electrons. As we will see below, some exist naturally as a consequence of lattice symmetry and some can be made to occur by tuning the microstructures of a phononic/photonic crystal. Although quite a bit of effort has been devoted to this topic recently, some fundamental questions remain unanswered. For example, the physical origin of linear dispersions of a Dirac or Dirac-like cone in classical waves is not well understood. Specifically, many authors assumed that a Dirac cone can always be described by the Dirac equation with two degenerate Bloch states at the $K$ point as the basis of the spinors. But this has only been shown explicitly in the nearly-free-photon approximation, ${ }^{2}$ not for a realistic phononic/photonic crystal, where Bloch states are the results of multiple scatterings. For graphene, the basis functions of the spinors are the atomic wave functions of two equivalent lattice sites in the unit cell of a honeycomb lattice. However, for a Dirac point in phononic/photonic crystals, this very fundamental information is still unknown to us thus far. Also, there exists no general theory that allows us to derive the linear slopes of a Dirac cone from first principles. Finally, it is not clear whether the Dirac-like cone dispersion at the $\Gamma$ point can also be described by the Dirac equation, although this has been widely assumed in the literature. ${ }^{13}$ The answer to this question is important because it is known that the Dirac equation leads to a Berry phase which in turn gives rise to antilocalization properties as found in disordered graphene.

To answer all the above questions, in this paper we propose a theory which generalizes the $\vec{k} \cdot \vec{p}$ method of electrons to classical waves, to study from the first principles the origin of Dirac/Dirac-like cones in phononic/photonic crystals. The $\vec{k} \cdot \vec{p}$ method is a perturbative method which takes the set of eigenfunctions at a particular symmetry point of interest as the unperturbed basis to study the eigenstates in the vicinity of that point. These eigenfunctions take account of all multiple scatterings in the phononic/photonic crystal and can be exactly determined. Furthermore, the result obtained from the method is valid independent of the details of the field distribution of the eigenmode, as it makes no assumption on whether the energy is concentrated in the host or in the embedding medium. Thus, the theory can accurately predict the slopes of linear dispersions at various symmetry points, independent of frequency and lattice structure. We note that the 
physical properties of Dirac-like systems can be discussed with effective medium theories only if the systems satisfy certain conditions. For example, the Dirac-like point should come from monopole and dipole degeneracy in two-dimensional (2D) photonic systems. ${ }^{10,11}$ The formulation here is more general and is valid even if effective medium theory does not apply. The reduced Hamiltonian constructed from the theory reveals that only Dirac cones can be mapped into the form of a massless Dirac Hamiltonian, and the basis functions of the spinors can be determined from the reduced Hamiltonian through some nontrivial unitary transformation. Furthermore, we show that such Dirac cones possess a Berry phase of $-\pi$ and, therefore, give rise to antilocalization effects. On the other hand, the Dirac-like cones with triple degeneracy cannot be mapped into the massless Dirac Hamiltonian and carry no Berry phase, and therefore, are expected to exhibit normal localization behaviors rather than antilocalization in the presence of disorder.

\section{METHODOLOGY}

We consider the following acoustic wave equation in a periodic structure:

$$
\nabla \cdot\left[\frac{1}{\rho_{r}(\vec{r})} \nabla p\right]=-\frac{\omega^{2}}{c_{1}^{2}} \cdot \frac{p}{B_{r}(\vec{r})},
$$

where $p$ is the pressure, $\rho_{r}(\vec{r})=\rho(\vec{r}) / \rho_{1}$ and $B_{r}(\vec{r})=B(\vec{r}) / B_{1}$ are the relative mass density and bulk modulus, respectively, and $c_{1}=\sqrt{B_{1} / \rho_{1}}$ is the speed of sound in the host. Our task is to find the origin of linear dispersions near a Dirac or Dirac-like cone located at some particular high-symmetry point $\vec{k}_{0}$ with frequency $\omega_{0}$, independent of the origin of the degeneracy. The approach is similar to the well-known $\vec{k} \cdot \vec{p}$ method for electrons. Although the method developed below is for phononic crystals, it is also applicable to 2D photonic crystals by using mapping of variables. Take the transverse magnetic (TM) polarization with $\vec{E}=\left(0,0, E_{z}\right)$ as an example; by making the following substitutions in Eq. (1)- $p \Leftrightarrow E_{z}$, $\rho_{r} \Leftrightarrow \mu_{r}, \frac{1}{B_{r}} \Leftrightarrow \varepsilon_{r}$-we will arrive at

$$
\nabla \cdot\left[\frac{1}{\mu_{r}(\vec{r})} \nabla E_{z}\right]=-\frac{\omega^{2}}{c^{2}} \cdot \varepsilon_{r}(\vec{r}) E_{z},
$$

which is exactly the wave equation satisfied by the TM polarization. The mapping to transverse electric (TE) polarization with $\vec{H}=\left(0,0, H_{z}\right)$ can also be made in a similar way.

Assuming that all the Bloch states at the $\vec{k}_{0}$ point are known, ${ }^{21}$ i.e., Bloch wave functions $\psi_{n \vec{k}_{0}}(\vec{r})=e^{i \overrightarrow{k_{0}} \cdot \vec{r}} u_{n \vec{k}_{0}}(\vec{r})$ and eigenfrequencies $\omega_{n 0}$, where " $n$ " denotes the band index, we can write the Bloch state at a wave vector $\vec{k}$ near $\vec{k}_{0}$ as

$$
\psi_{n \vec{k}}(\vec{r})=u_{n \vec{k}}(\vec{r}) e^{i \vec{k} \cdot \vec{r}}=\sum_{j} A_{n j}(\vec{k}) e^{i\left(\vec{k}-\vec{k}_{0}\right) \cdot \vec{r}} \psi_{j \vec{k}_{0}}(\vec{r}),
$$

where the unknown periodic functions $u_{n \vec{k}}(\vec{r})$ have been expressed as linear combinations of $u_{j \vec{k}_{0}}(\vec{r})$. Substituting Eq. (2) into Eq. (1), we obtain

$$
\begin{aligned}
& \sum_{j} A_{n j}(\vec{k}) e^{i\left(\vec{k}-\vec{k}_{0}\right) \cdot \vec{r}} \\
& \quad \times\left\{\frac{\left(\omega_{n \vec{k}}^{2}-\omega_{j 0}^{2}\right) \psi_{j \vec{k}_{0}}(\vec{r})}{c_{1}^{2} B_{r}(\vec{r})}+\frac{2 i\left(\vec{k}-\vec{k}_{0}\right) \cdot \nabla \psi_{j \vec{k}_{0}}(\vec{r})}{\rho_{r}(\vec{r})}\right. \\
& \left.\quad+\left[i\left(\vec{k}-\vec{k}_{0}\right) \cdot \nabla \frac{1}{\rho_{r}(\vec{r})}\right] \psi_{j \vec{k}_{0}}(\vec{r})-\frac{\left(\vec{k}-\vec{k}_{0}\right)^{2} \psi_{j \vec{k}_{0}}(\vec{r})}{\rho_{r}(\vec{r})}\right\}=0 .
\end{aligned}
$$

Utilizing the orthonormal property ${ }^{22}$ of the basis functions $\psi_{j \vec{k}_{0}}(\vec{r})$, i.e., $\frac{(2 \pi)^{2}}{\Omega} \int_{\text {unit cell }} \psi_{l \vec{k}_{0}}^{*}(\vec{r}) \frac{1}{B_{r}(\vec{r})} \psi_{j \vec{k}_{0}}(\vec{r}) d \vec{r}=\delta_{l j}$, where $\Omega$ is the area of a unit cell, Eq. (3) can be written as

$$
\sum_{j}\left[\frac{\omega_{j 0}^{2}-\omega_{n \vec{k}}^{2}}{c_{1}^{2}} \delta_{l j}-P_{l j}(\vec{k})\right] A_{n j}(\vec{k})=0,
$$

where

$$
P_{l j}(\vec{k})=\left(\vec{k}-\vec{k}_{0}\right) \cdot \vec{p}_{l j}-\left(\vec{k}-\vec{k}_{0}\right)^{2} q_{l j},
$$

with

$$
\begin{aligned}
\vec{p}_{l j}= & i \frac{(2 \pi)^{2}}{\Omega} \int_{\text {unit cell }} \psi_{\vec{k}_{0}}^{*}(\vec{r}) \\
& \times\left\{\frac{2 \nabla \psi_{j \vec{k}_{0}}(\vec{r})}{\rho_{r}(\vec{r})}+\left[\nabla \frac{1}{\rho_{r}(\vec{r})}\right] \psi_{j \vec{k}_{0}}(\vec{r})\right\} d \vec{r},
\end{aligned}
$$

and

$$
q_{l j}=\frac{(2 \pi)^{2}}{\Omega} \int_{\text {unit cell }} \psi_{l \vec{k}_{0}}^{*}(\vec{r}) \frac{1}{\rho_{r}(\vec{r})} \psi_{j \vec{k}_{0}}(\vec{r}) d \vec{r} .
$$

It is easy to show that the matrices $\vec{p}_{l j}$ and $q_{l j}$ are Hermitian, i.e., $\vec{p}_{j l}^{*}=\vec{p}_{l j}$ and $q_{j l}^{*}=q_{l j}$. Equation (4) has nontrivial solutions only when the following secular equation is satisfied:

$$
\operatorname{det}\left|H-\frac{\omega_{n \vec{k}}^{2}-\omega_{j 0}^{2}}{c_{1}^{2}} I\right|=0,
$$

where $H$ is the reduced Hamiltonian with matrix elements

$$
H_{l j}=-P_{l j} .
$$

To obtain the dispersion relation $\omega_{n \vec{k}}(\vec{k})$ from Eq. (8), one has to incorporate all Bloch states at $\vec{k}_{0}$. However, as we are interested in linear dispersions of a Dirac/Dirac-like cone, we only have to consider the degenerate states at the Dirac/Dirac-like point in the summation of Eq. (2). Other bands only contribute to the quadratic term $\left|\vec{k}-\vec{k}_{0}\right|^{2}$ in the dispersion relation. This greatly reduces the dimension of the matrix $H$, and makes Eq. (8) analytically solvable. The analytic solution to Eq. (8) for small $\Delta k \equiv\left|\vec{k}-\vec{k}_{0}\right|$ can be expressed as

$$
\frac{\Delta \omega_{\beta}}{\Delta k}=\gamma_{\beta} c_{1}+O(\Delta k) ; \quad \beta=1,2, \ldots, s,
$$

where $\Delta \omega_{\beta} \equiv \omega_{\beta \vec{k}}-\omega_{0}$, with $\omega_{0}$ and $s$ being, respectively, the frequency and number of degenerate Bloch states at the 
Dirac/Dirac-like point. Here we have approximated $\omega_{0}^{2}-\omega_{\beta \vec{k}}^{2}$ by $-2 \omega_{0} \Delta \omega_{\beta}$. The linear slopes $\gamma_{\beta}$ are determined by $\vec{p}_{l j}$ only, and $q_{l j}$ contributes to the $\Delta k^{2}$ term. Thus, the slope of the linear dispersion is proportional to the strength of the coupling between the degenerate Bloch states $\psi_{\vec{l} \vec{k}_{0}}$ and $\psi_{j \vec{k}_{0}}$, as shown in Eq. (6). Therefore, it is clear that the coupling of the degenerate Bloch states leads to the frequency repulsion effect, which in turn gives rise to linear dispersions of a Dirac/Dirac-like cone. It should be pointed out that the above perturbative approach is exact to the first order in $\Delta k^{23}$ Thus, the existence of linear dispersions requires both the degeneracy of Bloch states at some high-symmetry point $\vec{k}_{0}$ and the corresponding $\vec{p}_{l j} \neq 0$, independent of whether the degeneracy is accidental or due to lattice symmetry. The matrix elements of $\vec{p}_{l j}$ and $q_{l j}$ can be easily evaluated by performing numerical integration of Eqs. (6) and (7) using the knowledge of degenerate Bloch wave functions at $\vec{k}_{0}$. There are various ways to obtain the Bloch wave functions for phononic/photonic crystals numerically. In this work, we adopted the COMSOL Multiphysics, a commercial package based on the finite-element method.

\section{RESULTS AND DISCUSSIONS}

\section{A. Physical system}

Figure 1(a) shows the band structures of a triangular array of iron cylinders, with radii of $0.3203 a$ ( $a$ is the lattice constant), embedded in a water host calculated by COMSOL. There exist two points, marked by " $A$ " and " $B$ ", at which linear dispersions are found. The mass densities of iron and water are $\rho_{2}=7670 \mathrm{~kg} / \mathrm{m}^{3}$ and $\rho_{1}=1000 \mathrm{~kg} / \mathrm{m}^{3}$, respectively, and the corresponding longitudinal wave velocities are $c_{2}=6010 \mathrm{~m} / \mathrm{s}$ for iron and $c_{1}=1490 \mathrm{~m} / \mathrm{s}$ for water. Since the longitudinal

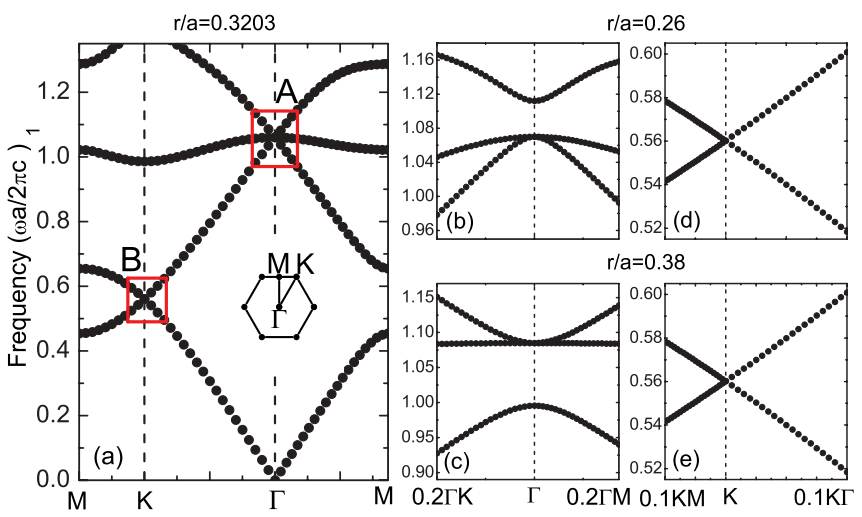

FIG. 1. (Color online) (a) Band structures of a 2D phononic crystal consisting of a triangular lattice of iron rods embedded in a water host, with a rod radius $r / a=0.3203$, where $a$ is the lattice constant. Point $A$ is a Dirac-like point while point $B$ is a Dirac point. The inset shows the Brillouin zone. (b), (c) Closeup of bandstructures around the $\Gamma$ point for different rod radii, with $r / a=0.26$ in (b), and $r / a=0.38$ in (c), respectively. (d), (e) Closeup of band structures around the $K$ point for different rod radii, with $r / a=0.26$ in (d), and $r / a=0.38$ in (e), respectively. The red boxes in Fig. 1(a) mark the areas from where Figs. 1(b)-1(e), 2(d), and 3(c) are extended. wave velocity contrast between iron and water is large, the shear modes inside the iron cylinders are not important and can be ignored. ${ }^{24}$ This has simplified our calculations.

\section{B. Dirac-like cone at the Brillouin zone center}

\section{The reduced Hamiltonian and slopes of linear dispersions}

Point $A$ shows a Dirac-like cone created by accidental degeneracy of three Bloch states at the $\Gamma$ point $\left(\vec{k}_{0}=0\right)$, where the frequencies for the single Bloch state, $\psi_{1 \Gamma}$, and the doubly degenerate Bloch states, $\psi_{2 \Gamma}$ and $\psi_{3 \Gamma}$, are tuned deliberately to coincide by adjusting the radii of the iron cylinders. Figures 1(b) and 1(c) illustrate the band structures near the $\Gamma$ point for smaller and larger iron cylinders, i.e., $r / a=0.26$ and 0.38 , respectively. Both figures show clearly the separated single mode and doubly degenerate modes. In Fig. 1 we have used dimensionless units for frequency, $\omega a / 2 \pi c_{1}$, and the radii of the iron cylinders, $r / a$. The three degenerate Bloch states $\psi_{1 \Gamma}, \psi_{2 \Gamma}$, and $\psi_{3 \Gamma}$ at point $A$ when $r / a$ is tuned to 0.3203 are shown in Figs. 2(a)-2(c), respectively. To the linear order in $k$, the reduced Hamiltonian near the point $A$ has the following form:

$$
H=\left(\begin{array}{lcc}
0 & i \vec{k} \cdot \vec{L}_{12} & i \vec{k} \cdot \vec{L}_{13} \\
-i \vec{k} \cdot \vec{L}_{12} & 0 & 0 \\
-i \vec{k} \cdot \vec{L}_{13} & 0 & 0
\end{array}\right),
$$

where $\vec{L}_{l j}=-i \vec{p}_{l j}$, and $\vec{L}_{12}$ and $\vec{L}_{13}$ are two real vectors in the $x$ - $y$ plane. Numerically we find $\vec{L}_{12}=\frac{1}{a}(-9.9847,2.2755)$ and $\vec{L}_{13}=\frac{1}{a}(-2.2755,-9.9847)$. It is interesting to see that $\vec{L}_{12}$ and $\vec{L}_{13}$ have the same length $s / a$, and are perpendicular
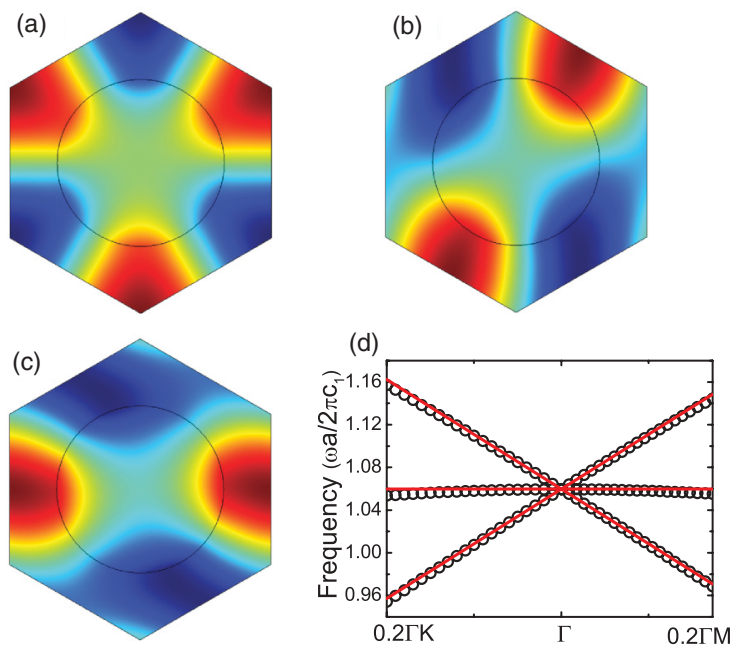

FIG. 2. (Color online) (a)-(c) Pressure field distributions of three degenerate Bloch states, $\psi_{1 \Gamma}, \psi_{2 \Gamma}$, and $\psi_{3 \Gamma}$, respectively, at point $A$ as indicated in Fig. 1. Dark red and dark blue denote the positive and negative maxima, respectively, which imply that the wave energy is mainly concentrated in the host medium. (d) Black circles show the band structure near point $A$ obtained by finite-element calculations. Red curves show the results predicted by Eqs. (8) and (11). 
to each other, i.e., $\left|\vec{L}_{12}\right|=\left|\vec{L}_{13}\right|=s / a$ and $\vec{L}_{12} \cdot \vec{L}_{13}=0$. These properties are actually required by the isotropy of the linear dispersion. ${ }^{25,26}$ By using Eq. (8), we find $\Delta \omega / k=$ $\pm s c_{1}^{2} /\left(2 \omega_{0} a\right)$ and 0 . From the length of $\vec{L}_{12}$ or $\vec{L}_{13}$, we obtain $s=10.241$. With the frequency at point $A, \omega_{0} a /\left(2 \pi c_{1}\right)=$ 1.06, as shown in Fig. 1(a), we find $\Delta \omega / k= \pm 0.769 c_{1}$ and 0 . Obviously, the first two values correspond to the two linear dispersions and the third one corresponds to the flat band shown near the point $A$. These results agree excellently with the band structure calculations as can be seen from Fig. 2(d). It is worth mentioning that if wave energy is localized within the scatterers, then the slopes of the Dirac-like cone can also be calculated by using a tight-binding model proposed in Ref. 12. However, our theory does not have such restrictions as shown in Fig. 2, in which the wave energy is mainly concentrated in the host.

\section{Symmetry analysis}

According to our theory developed in Sec. II, the existence of linear dispersions requires that the matrix elements $\vec{p}_{l j} \equiv$ $i \vec{L}_{l j} \equiv i\left\langle\psi_{l \vec{k}_{0}}|\vec{L}| \psi_{j \vec{k}_{0}}\right\rangle$ be nonzero. From group theory, the condition of whether $\left\langle\psi_{l \vec{k}_{0}}|\vec{L}| \psi_{j \vec{k}_{0}}\right\rangle$ is zero turns out to be whether the direct product of the irreducible representations of $\psi_{l \vec{k}_{0}}, \vec{L}$, and $\psi_{j \vec{k}_{0}}$ contains $A_{1}$, the fully symmetrical representation. Any Bloch state at the $\Gamma$ point of a triangular lattice is an irreducible representation of the $C_{6 v}$ group. As shown in Fig. 2, the triply degenerate Bloch states are composed of one single state, $\psi_{1 \Gamma}$, corresponding to the $B_{2}$ representation, and doubly degenerate states, $\psi_{2 \Gamma}$ and $\psi_{3 \Gamma}$, transforming as the $E_{2}$ representation. The operator $\vec{L}$ transforms like a vector ${ }^{27}$ and corresponds to the $E_{1}$ irreducible representation in $C_{6 v}$. Since the direct product $B_{2} \otimes E_{1} \otimes E_{2}$ contains $A_{1}$, we have a nonzero $\left\langle\psi_{1 \Gamma}|L| \psi_{2 \Gamma, 3 \Gamma}\right\rangle$, which implies the existence of linear dispersions around point $A$.

\section{The Berry phase}

From Eq. (11), we find the following Bloch eigenfunctions near point $A$ :

$$
\begin{gathered}
\Psi_{+}(\vec{k})=\frac{1}{\sqrt{2}}\left(\begin{array}{c}
1 \\
i \hat{k} \cdot \hat{L}_{12} \\
i \hat{k} \cdot \hat{L}_{13}
\end{array}\right) e^{i \vec{k} \cdot \vec{r}}, \\
\Psi_{-}(\vec{k})=\frac{1}{\sqrt{2}}\left(\begin{array}{c}
1 \\
-i \hat{k} \cdot \hat{L}_{12} \\
-i \hat{k} \cdot \hat{L}_{13}
\end{array}\right) e^{i \vec{k} \cdot \vec{r}}, \\
\Psi_{0}(\vec{k})=\frac{1}{\sqrt{2}}\left(\begin{array}{c}
0 \\
-i \hat{k} \cdot \hat{L}_{13} \\
i \hat{k} \cdot \hat{L}_{12}
\end{array}\right) e^{i \vec{k} \cdot \vec{r}},
\end{gathered}
$$

for $\Delta \omega>0, \Delta \omega<0$, and $\Delta \omega=0$, respectively, where $\hat{k} \equiv$ $\frac{\vec{k}}{k}, \hat{L}_{12} \equiv \frac{\vec{L}_{12}}{\left|\vec{L}_{12}\right|}$, and $\hat{L}_{13} \equiv \frac{\vec{L}_{13}}{\left|\vec{L}_{13}\right|}$ are the unit vectors along the $\vec{k}$, $\vec{L}_{12}$, and $\vec{L}_{13}$ directions, respectively. From Eqs. (12) and (13), we can calculate the Berry phase ${ }^{28}$ of the Dirac-like cone:

$$
\Gamma_{ \pm}=i \oint\left\langle\Psi_{ \pm}(\vec{k})\left|\nabla_{\vec{k}}\right| \Psi_{ \pm}(\vec{k})\right\rangle \cdot d \vec{k}
$$

Let us take $\Psi_{+}(\vec{k})$ as an example. We can write $\hat{L}_{12}=$ $-u \hat{x}+v \hat{y}$ and $\hat{L}_{13}=-v \hat{x}-u \hat{y}$, with $u^{2}+v^{2}=1$. Substituting Eq. (12) into Eq. (15), we find

$$
\begin{aligned}
\Gamma_{+}= & i \oint\left\langle\Psi_{+}(\vec{k})\left|\nabla_{\vec{k}}\right| \Psi_{+}(\vec{k})\right\rangle \cdot d \vec{k} \\
= & \frac{i}{2} \oint\left[1,-\frac{i}{k}\left(-u k_{x}+v k_{y}\right),-\frac{i}{k}\left(-v k_{x}-u k_{y}\right)\right] \\
& \times\left\{\left[\begin{array}{c}
0 \\
\frac{i}{k}(-u \hat{x}+v \hat{y}) \\
\frac{i}{k}(-v \hat{x}-u \hat{y})
\end{array}\right]+\left[\begin{array}{c}
1 \\
\frac{i}{k}\left(-u k_{x}+v k_{y}\right) \\
\frac{i}{k}\left(-v k_{x}-u k_{y}\right)
\end{array}\right] i \vec{r}\right\} \cdot d \vec{k} \\
= & \frac{i}{2} \oint\left[\frac{1}{k^{2}}\left(k_{x} d k_{x}+k_{y} d k_{y}\right)\right]+\frac{i}{2} \oint 2 i \vec{r} \cdot d \vec{k},
\end{aligned}
$$

in which the second term on the right-hand side is obviously zero, while the first term can be simplified as

$$
\begin{aligned}
& \frac{i}{2} \oint\left[\frac{1}{k^{2}}\left(k_{x} d k_{x}+k_{y} d k_{y}\right)\right] \\
& =\frac{i}{2 k^{2}} \oint d \vec{k} \cdot \vec{A} \\
& \quad=\frac{i}{2 k^{2}} \int_{\sigma}(\nabla \times \vec{A}) \cdot d \vec{\sigma}=0,
\end{aligned}
$$

since $\vec{A}=k_{x} \hat{x}+k_{y} \hat{y}$ so that $\nabla \times \vec{A}=0$. Finally, we arrive at $\Gamma_{+}=0$. The same result is obtained if we use $\Psi_{-}(\vec{k})$ in Eq. (16). Thus, we find that the Berry phase for the Dirac-like cone is zero. This implies that the cone found at the $\Gamma$ point is actually not a Dirac cone. It should be pointed out that the presence of a linear dispersion is not the only property of a Dirac cone. The eigenstates on the Dirac cone should satisfy the massless Dirac equation and carry a Berry phase $\pm \pi$. The reduced Hamiltonian found in Eq. (11) shows a $3 \times 3$ matrix representation, and, therefore, its eigenstates are in the forms of Eqs. (12) and (13). These states cannot be the solutions of the massless Dirac Hamiltonian, which has a $2 \times 2$ matrix representation. This again points to the fact that the cone at the $\Gamma$ point is not a pure Dirac cone. Here, we call it a Dirac-like cone.

It is important to note that although the dispersion here also has two cones touching at one point as in the case of graphene, the existence of a third degree of freedom changes the physics. Some papers in the literature (see, e.g., Ref. 13) used the Dirac equation to describe the transport properties of these systems and such assumption can lead to an erroneous conclusion in the presence of disorder as the zero Berry phase implies that a Dirac-like cone exhibits normal localization behavior rather than antilocalization.

\section{Dirac cone at the Brillouin zone boundary}

\section{The reduced Hamiltonian}

Point $B$ in Fig. 1(a) represents a Dirac point where two bands meet at the $K$ point. These two bands are always degenerate at the $K$ point of a triangular/honeycomb lattice, 

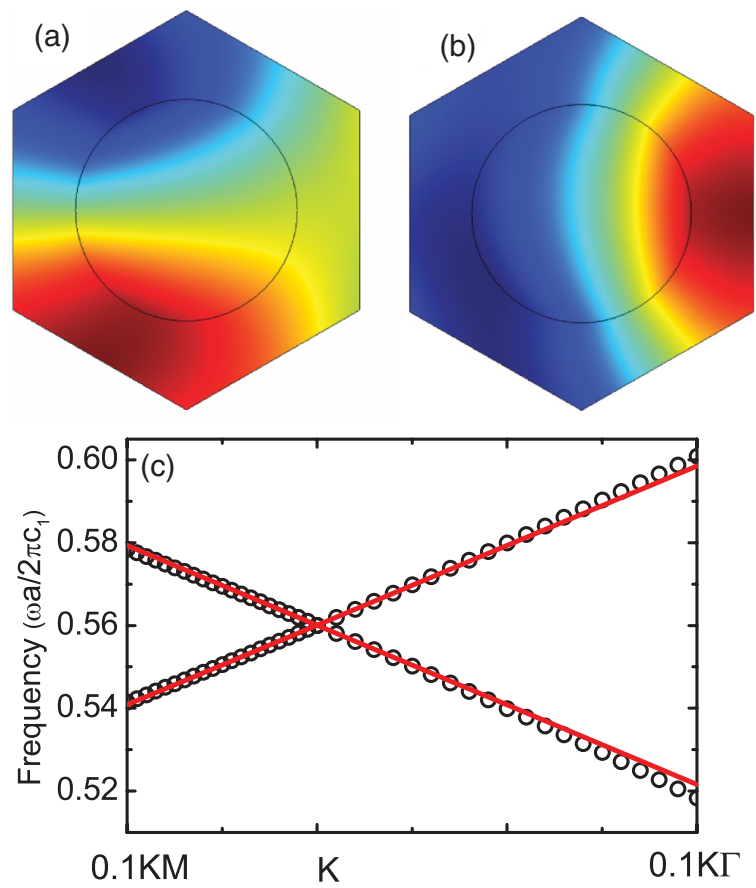

FIG. 3. (Color online) (a), (b) Pressure field distributions of two degenerate Bloch states, $\psi_{1 K}$ and $\psi_{2 K}$, respectively, at point $B$ as indicated in Fig. 1. Dark red and dark blue denote the positive and negative maxima, respectively. (c) Black circles show the band structures near point B obtained by finite-element calculations. Red curves show the results predicted by Eqs. (8) and (18).

independent of the radii of the scatterers. This is a result of crystal symmetry and is called the "deterministic degeneracy." In Figs. 1(d) and 1(e), we plot the dispersions around the $K$ point for two different radii, i.e., $r / a=0.26$ and 0.38 . It is clearly seen that the degeneracy remains. The two degenerate Bloch states $\psi_{1 K}$ and $\psi_{2 K}$ are shown in Figs. 3(a) and 3(b), respectively. The reduced Hamiltonian has the following form:

$$
\begin{aligned}
H & =\Delta \vec{k} \cdot\left(\begin{array}{lr}
\vec{p}_{11} & \operatorname{Re} \vec{p}_{12}+i \operatorname{Im} \vec{p}_{12} \\
\operatorname{Re} \vec{p}_{12}-i \operatorname{Im} \vec{p}_{12} & -\vec{p}_{11}
\end{array}\right) \\
& =\left(\Delta \vec{k} \cdot \operatorname{Re} \vec{p}_{12}\right) \sigma_{1}-\left(\Delta \vec{k} \cdot \operatorname{Im} \vec{p}_{12}\right) \sigma_{2}+\left(\Delta \vec{k} \cdot \vec{p}_{11}\right) \sigma_{3} \\
& =d_{1} \sigma_{1}+d_{2} \sigma_{2}+d_{3} \sigma_{3}=\vec{d} \cdot \vec{\sigma}
\end{aligned}
$$

where $\vec{\sigma}=\left(\sigma_{1}, \sigma_{2}, \sigma_{3}\right)$ are the Pauli matrices and $\sigma_{i}$ are in the basis of $\left(\begin{array}{l}1 \\ 0\end{array}\right)=\psi_{1 K},\left(\begin{array}{l}0 \\ 1\end{array}\right)=\psi_{2 K} \cdot \vec{p}_{11}, \operatorname{Re} \vec{p}_{12}$, and $\operatorname{Im} \vec{p}_{12}$ are three real vectors in the $x-y$ plane. The vector $\vec{d}$ is a function of $\Delta \vec{k}$ with its 3 components given by

$$
\begin{gathered}
d_{1}=\Delta \vec{k} \cdot \operatorname{Re} \vec{p}_{12}=(\Delta k)_{x}\left(\operatorname{Re} \vec{p}_{12}\right)_{x}+(\Delta k)_{y}\left(\operatorname{Re} \vec{p}_{12}\right)_{y}, \\
d_{2}=-\Delta \vec{k} \cdot \operatorname{Im} \vec{p}_{12} \\
=-(\Delta k)_{x}\left(\operatorname{Im} \vec{p}_{12}\right)_{x}-(\Delta k)_{y}\left(\operatorname{Im} \vec{p}_{12}\right)_{y}, \\
d_{3}=\Delta \vec{k} \cdot \vec{p}_{11}=(\Delta k)_{x}\left(\vec{p}_{11}\right)_{x}+(\Delta k)_{y}\left(\vec{p}_{11}\right)_{y} .
\end{gathered}
$$

Thus

$$
\begin{aligned}
H= & (\Delta k)_{x}\left[\left(\operatorname{Re} \vec{p}_{12}\right)_{x} \sigma_{1}-\left(\operatorname{Im} \vec{p}_{12}\right)_{x} \sigma_{2}+\left(\vec{p}_{11}\right)_{x} \sigma_{3}\right] \\
& +(\Delta k)_{y}\left[\left(\operatorname{Re} \vec{p}_{12}\right)_{y} \sigma_{1}-\left(\operatorname{Im} \vec{p}_{12}\right)_{y} \sigma_{2}+\left(\vec{p}_{11}\right)_{y} \sigma_{3}\right] \\
= & (\Delta k)_{x}\left(\vec{\alpha}_{x} \cdot \vec{\sigma}\right)+(\Delta k)_{y}\left(\vec{\alpha}_{y} \cdot \vec{\sigma}\right)
\end{aligned}
$$

where

$$
\begin{aligned}
\vec{\alpha}_{x} & =\left[\left(\operatorname{Re} \vec{p}_{12}\right)_{x},-\left(\operatorname{Im} \vec{p}_{12}\right)_{x},\left(\vec{p}_{11}\right)_{x}\right], \\
\vec{\alpha}_{y} & =\left[\left(\operatorname{Re} \vec{p}_{12}\right)_{y},-\left(\operatorname{Im} \vec{p}_{12}\right)_{y},\left(\vec{p}_{11}\right)_{y}\right],
\end{aligned}
$$

are two three-dimensional real vectors in the pseudo-spin space. The isotropy of the Dirac cone requires again that $\vec{\alpha}_{x}$ and $\vec{\alpha}_{y}$ have the same length and are perpendicular to each other, i.e.,

$$
\begin{gathered}
\vec{\alpha}_{x} \cdot \vec{\alpha}_{y}=0 \\
\left|\vec{\alpha}_{x}\right|=\left|\vec{\alpha}_{y}\right|=\frac{s}{a},
\end{gathered}
$$

with

$$
\begin{aligned}
\frac{s}{a} & =\frac{|\vec{d}|}{\Delta k}=\sqrt{\left(\operatorname{Re} \vec{p}_{12}\right)_{x}^{2}+\left(\operatorname{Im} \vec{p}_{12}\right)_{x}^{2}+\left(\vec{p}_{11}\right)_{x}^{2}} \\
& =\sqrt{\left(\operatorname{Re} \vec{p}_{12}\right)_{y}^{2}+\left(\operatorname{Im} \vec{p}_{12}\right)_{y}^{2}+\left(\vec{p}_{11}\right)_{y}^{2}} .
\end{aligned}
$$

Numerical results given in the following section confirm the equalities given in Eqs. (25)-(27). Equation (18) can be mapped into the Dirac Hamiltonian by applying three successive rotations in the pseudo-spin space involving three Euler angles. Specifically, we first rotate around the $\hat{3}$ axis to move the $\hat{1}$ axis into the plane spanned by $\vec{\alpha}_{x}$ and $\vec{\alpha}_{y}$. Then we rotate around the $\hat{1}^{\prime}$ axis to move $\hat{2}^{\prime}$ into the plane spanned by $\vec{\alpha}_{x}$ and $\vec{\alpha}_{y}$. Finally, we rotate around the $\hat{3}^{\prime \prime}$ axis so that $\hat{1}^{\prime \prime}$ and $\hat{2}^{\prime \prime}$ coincide with $\vec{\alpha}_{x}$ and $\vec{\alpha}_{y}$, respectively. After these rotations, we can write $\vec{\sigma}=\sigma_{x} \hat{1}^{\prime \prime}+\sigma_{y} \hat{2}^{\prime \prime}+\sigma_{z} \hat{3}^{\prime \prime}$ and Eq. (18) becomes

$$
H=\frac{s}{a}\left[(\Delta k)_{x} \sigma_{x}+(\Delta k)_{y} \sigma_{y}\right],
$$

where $\sigma_{x, y}=\frac{\vec{\alpha}_{x, y}}{s / a} \cdot \vec{\sigma}$ are the new Pauli matrices in the transformed pseudo-spin space. As shown in Eq. (28), the Hamiltonian can be cast into the Dirac form.

\section{Gradients of linear dispersions}

The matrix elements $\vec{p}_{l j}$ and $q_{l j}$ can be evaluated using the Bloch states $\psi_{j K}(\vec{r}),(j=1,2)$ obtained from COMSOL Multiphysics. From the numerical data we obtain

$$
\begin{gathered}
\vec{p}_{11}=\frac{1}{a}(4.0068,-0.6585), \\
\operatorname{Re} \vec{p}_{12}=\frac{1}{a}(-0.6615,-4.0017), \\
\operatorname{Im} \vec{p}_{12}=\frac{1}{a}(-0.03944,0.2160), \\
s=\sqrt{4.0068^{2}+0.6615^{2}+0.03944^{2}}=4.0612 .
\end{gathered}
$$

It is easy to show that the components of the three vectors $\vec{p}_{11}, \operatorname{Re} \vec{p}_{12}$, and $\operatorname{Im} \vec{p}_{12}$ satisfy the following 
relations:

$$
\begin{aligned}
& \left(\operatorname{Re} \vec{p}_{12}\right)_{x}^{2}+\left(\operatorname{Im} \vec{p}_{12}\right)_{x}^{2}+\left(\vec{p}_{11}\right)_{x}^{2} \\
& \quad=\left(\operatorname{Re} \vec{p}_{12}\right)_{y}^{2}+\left(\operatorname{Im} \vec{p}_{12}\right)_{y}^{2}+\left(\vec{p}_{11}\right)_{y}^{2},
\end{aligned}
$$

$\left(\operatorname{Re} \vec{p}_{12}\right)_{x}\left(\operatorname{Re} \vec{p}_{12}\right)_{y}+\left(\operatorname{Im} \vec{p}_{12}\right)_{x}\left(\operatorname{Im} \vec{p}_{12}\right)_{y}+\left(\vec{p}_{11}\right)_{x}\left(\vec{p}_{11}\right)_{y}=0$,

which obviously agree with $\vec{\alpha}_{x} \cdot \vec{\alpha}_{y}=0$ and $\left|\vec{\alpha}_{x}\right|=\left|\vec{\alpha}_{y}\right|=\frac{s}{a}$, as specified previously in Eqs. (25) and (26).

As mentioned in Sec. II, our theory is exact to the first order in $\Delta k$ (Ref. 23) so that it can correctly predict the linear slopes of the Dirac cone. By solving the secular equation, we find

$$
-\frac{2 \omega_{0} \Delta \omega_{\beta}}{c_{1}^{2}}= \pm \frac{s}{a} \Delta k
$$

with $s=4.0612$. Thus, the slopes of the linear dispersions are

$$
\frac{\Delta \omega_{\beta}}{\Delta k}=\gamma_{\beta} c_{1},
$$

where $\gamma_{\beta}= \pm \frac{s c_{1}}{2 \omega_{0} a}= \pm 0.577$, with the dimensionless frequency $\frac{\omega_{0} a}{2 \pi c_{1}}=0.560$. The predicted slopes of the linear dispersions near point $B$, i.e., $\pm 0.577 c_{1}$, are shown as red curves in Fig. 3(c), which agree excellently with the band structure calculations.

\section{Symmetry analysis}

The existence of linear dispersions near point $B$ can also be understood from group analysis. The point group at the $K$ point of a triangular lattice has $C_{3 v}$ symmetry. The doubly degenerate Bloch states $\psi_{1 K}$ and $\psi_{2 K}$ shown in Fig. 3, transform as the $E$ irreducible representation. The existence of such a doublet is governed by the $C_{3 v}$ symmetry regardless of the radii of the inclusions. Since the operator $\vec{L}$ transforms as the $E$ representation in the $C_{3 v}$ group and the direct product $E \otimes E \otimes E$ again contains $A_{1}$, we have linear dispersions. Thus, the existence of a Dirac cone at the $K$ point is deterministic.

\section{The Berry phase}

The Berry phase can be evaluated by using the eigenfunctions of the reduced Hamiltonian given in Eq. (18). We assume that the angles between the vector $\vec{p}_{11}$ and the vectors $\Delta \vec{k}$, $\operatorname{Re} \vec{p}_{12}, \operatorname{Im} \vec{p}_{12}$ are $\varphi, \alpha, \beta$, respectively. Thus

$$
\begin{gathered}
d_{1}=\Delta \vec{k} \cdot \operatorname{Re} \vec{p}_{12}=\Delta k\left|\operatorname{Re} \vec{p}_{12}\right| \cos (\alpha-\varphi), \\
d_{2}=-\Delta \vec{k} \cdot \operatorname{Im} \vec{p}_{12}=-\Delta k\left|\operatorname{Im} \vec{p}_{12}\right| \cos (\beta-\varphi), \\
d_{3}=\Delta \vec{k} \cdot \vec{p}_{11}=\Delta k\left|\vec{p}_{11}\right| \cos \varphi .
\end{gathered}
$$

From Eq. (18), we find the following forms for the two Bloch states near point $B$ :

$$
\Psi_{+}(\Delta \vec{k})=\frac{1}{\sqrt{2}}\left(\begin{array}{c}
\sqrt{1-\Delta \hat{k} \cdot \vec{M}_{11}} \\
\frac{-\Delta \hat{k} \cdot \vec{M}_{12}^{*}}{\sqrt{1-\Delta \hat{k} \cdot \vec{M}_{11}}}
\end{array}\right) e^{i \Delta \vec{k} \cdot \vec{r}} \quad(\Delta \omega>0),
$$

$$
\Psi_{-}(\Delta \vec{k})=\frac{1}{\sqrt{2}}\left(\begin{array}{c}
\sqrt{1+\Delta \hat{k} \cdot \vec{M}_{11}} \\
\frac{\Delta \hat{k} \cdot \vec{M}_{12}^{*}}{\sqrt{1+\Delta \hat{k} \cdot \vec{M}_{11}}}
\end{array}\right) e^{i \Delta \vec{k} \cdot \vec{r}} \quad(\Delta \omega<0),
$$

where $\Delta \hat{k} \equiv \frac{\Delta \vec{k}}{\Delta k}$ is a unit vector along the $\Delta \vec{k}$ direction, and $\vec{M}_{11} \equiv \frac{\vec{p}_{11}}{s / a}$ and $\vec{M}_{12} \equiv \frac{\vec{p}_{12}^{*}}{s / a}$ are two dimensionless vectors. From this, we can calculate the Berry phase:

$$
\Gamma_{ \pm}=i \oint\left\langle\Psi_{ \pm}(\Delta \vec{k})\left|\nabla_{\Delta \vec{k}}\right| \Psi_{ \pm}(\Delta \vec{k})\right\rangle \cdot d(\Delta \vec{k}) .
$$

Let us take $\Psi_{+}(\Delta \vec{k})$ as an example, since

$$
\begin{gathered}
\Delta \hat{k} \cdot \vec{M}_{11}=\frac{\left|\vec{p}_{11}\right|}{s / a} \cos \varphi, \\
\Delta \hat{k} \cdot \vec{M}_{12}^{*}=\Delta \hat{k} \cdot \frac{\vec{p}_{12}^{*}}{s / a} \\
=\frac{\left|\operatorname{Re} \vec{p}_{12}\right|}{s / a} \cos (\alpha-\varphi)-i \frac{\left|\operatorname{Im} \vec{p}_{12}\right|}{s / a} \cos (\beta-\varphi),
\end{gathered}
$$

we have

$$
\begin{aligned}
& \Gamma_{+}=i \oint\left\langle\Psi_{+}(\Delta \vec{k})\left|\nabla_{\Delta \vec{k}}\right| \Psi_{+}(\Delta \vec{k})\right\rangle \cdot d(\Delta \vec{k})
\end{aligned}
$$

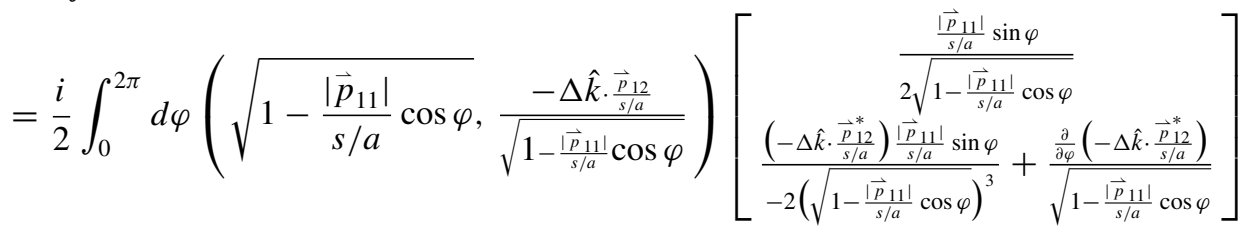

$$
\begin{aligned}
& =\frac{i}{4} \frac{\left|\vec{p}_{11}\right|}{s / a} \int_{0}^{2 \pi} d \varphi \sin \varphi-\frac{i}{4} \frac{\left|\vec{p}_{11}\right|}{s / a} \int_{0}^{2 \pi} d \varphi \frac{\left(\Delta \hat{k} \cdot \frac{\vec{p}_{12}}{s / a}\right)\left(\Delta \hat{k} \cdot \frac{\vec{p}_{12}^{*}}{s / a}\right) \sin \varphi}{\left(1-\frac{\left|\vec{p}_{11}\right|}{s / a} \cos \varphi\right)^{2}}+\frac{i}{2} \int_{0}^{2 \pi} d \varphi \frac{\left(\Delta \hat{k} \cdot \frac{\vec{p}_{12}}{s / a}\right) \frac{\partial}{\partial \varphi}\left(\Delta \hat{k} \cdot \frac{\vec{p}_{12}^{*}}{s / a}\right)}{1-\frac{\left|\vec{p}_{11}\right|}{s / a} \cos \varphi},
\end{aligned}
$$


in which the first two terms on the right side are zero, while the third term can be evaluated as

$$
\frac{i}{2} \int_{0}^{2 \pi} d \varphi \frac{\left(\Delta \hat{k} \cdot \frac{\vec{p}_{12}}{s / a}\right) \frac{\partial}{\partial \varphi}\left(\Delta \hat{k} \cdot \frac{\vec{p}_{12}^{*}}{s / a}\right)}{1-\frac{\left|\vec{p}_{11}\right|}{s / a} \cos \varphi}=-\frac{\sin (\alpha-\beta)}{2} \cdot \frac{\left|\operatorname{Re} \vec{p}_{12}\right|}{s / a} \cdot \frac{\left|\operatorname{Im} \vec{p}_{12}\right|}{s / a} \int_{0}^{2 \pi} \frac{d \varphi}{1-\frac{\left|\vec{p}_{11}\right|}{s / a} \cos \varphi}=-\pi .
$$

Using $\Psi_{-}(\vec{k})$, we obtain the same result. Therefore, we find that the Berry phase $\Gamma_{ \pm}=-\pi$ for the linear dispersions around point $B$. This result is consistent with the fact that Eq. (18) can be mapped into the Dirac Hamiltonian.

\section{CONCLUDING REMARKS}

To conclude, we have developed a first-principles theory to study the origin of Dirac/Dirac-like cone dispersions in phononic/photonic crystals. The theory can predict accurately the slopes of linear dispersions of a Dirac/Dirac-like cone at any symmetric point in a Brillouin zone, independent of frequency and lattice structure. Symmetry analysis based on the theory provides an easy and quick check of the existence of linear dispersion. The reduced Hamiltonian constructed from the theory shows that only the Dirac cones created by doubly degenerate Bloch states can be mapped into the massless Dirac Hamiltonian and carry a Berry phase. The absence of a Berry phase in Dirac-like cones implies normal localization behavior when disorder is introduced.

\section{ACKNOWLEDGMENTS}

We thank Professor Bradley A. Foreman, Professor K. T. Law, and Professor P. Sheng for stimulating discussions. This work was supported by the National Natural Science Foundation of China (Grant No. 10804086), the Ph.D. Programs Foundation of the Ministry of Education of China (Grant No. 200804861018), the Fundamental Research Funds for the Central Universities (Grant No. 2012ZZ0077), KAUST Start-up Package, and Hong Kong RGC (Grant No. 600311). *phjunmei@scut.edu.cn

${ }^{\dagger}$ ying.wu@kaust.edu.sa

${ }^{\ddagger}$ phchan@ust.hk

§phzzhang@ust.hk

${ }^{1}$ A. H. Castro Neto, F. Guinea, N. M. R. Peres, K. S. Novoselov, and

A. K. Geim, Rev. Mod. Phys. 81, 109 (2009).

${ }^{2}$ F. D. M. Haldane and S. Raghu, Phys. Rev. Lett. 100, 013904 (2008); Phys. Rev. A 78, 033834 (2008).

${ }^{3}$ R. A. Sepkhanov, Y. B. Bazaliy, and C. W. J. Beenakker, Phys. Rev. A 75, 063813 (2007); Opt. Commun. 281, 5267 (2008).

${ }^{4}$ X. Zhang, Phys. Rev. Lett. 100, 113903 (2008); X. Zhang and Z. Liu, ibid. 101, 264303 (2008); X. Zhang, Phys. Lett. A 372, 3512 (2008).

${ }^{5}$ M. Diem, T. Koschny, and C. M. Soukoulis, Physica B 405, 2990 (2010).

${ }^{6}$ Q. Liang, Y. Yan, and J. Dong, Opt. Lett. 36, 2513 (2011).

${ }^{7}$ O. Peleg, G. Bartal, B. Freedman, O. Manela, M. Segev, and D. N. Christodoulides, Phys. Rev. Lett. 98, 103901 (2007).

${ }^{8}$ T. Ochiai and M. Onoda, Phys. Rev. B 80, 155103 (2009).

${ }^{9}$ S. Bittner, B. Dietz, M. Miski-Oglu, P. Oria Iriarte, A. Richter, and F. Schafer, Phys. Rev. B 82, 014301 (2010).

${ }^{10}$ X. Huang, Y. Lai, Z. H. Hang, H. Zheng, and C. T. Chan, Nat. Mater. 10, 582 (2011).

${ }^{11}$ F. M. Liu, Y. Lai, X. Q. Huang, and C. T. Chan, Phys. Rev. B 84, 224113 (2011); Appl. Phys. Lett. 100, 071911 (2012).

${ }^{12}$ K. Sakoda, Opt. Express 20, 3898 (2012).

${ }^{13}$ L. G. Wang, Z. G. Wang, and S. Y. Zhu, Europhys. Lett. 86, 47008 (2009); Opt. Lett. 34, 1510 (2009); X. Chen, L. G. Wang, and C. F. Li, Phys. Rev. A 80, 043839 (2009); L. G. Wang, G. X. Li, and
S. Y. Zhu, Phys. Rev. B 81, 073105 (2010); M. Shen, L. X. Ruan, and X. Chen, Opt. Express 18, 12779 (2010); M. Shen, L. Ruan, X. Wang, J. Shi, and Q. Wang, Phys. Rev. A 83, 045804 (2011).

${ }^{14}$ K. Sakoda and H. Zhou, Opt. Express 18, 27371 (2010); 19, 13899 (2011).

${ }^{15}$ Y. D. Chong, X.-G. Wen, and M. Soljačić, Phys. Rev. B 77, 235125 (2008).

${ }^{16}$ W. Zhong and X. Zhang, Phys. Rev. A 84, 033826 (2011).

${ }^{17}$ F. Dreisow, M. Heinrich, R. Keil, A. Tunnermann, S. Nolte, S. Longhi, and A. Szameit, Phys. Rev. Lett. 105, 143902 (2010).

${ }^{18}$ S. H. Nam, J. Zhou, A. J. Taylor, and A. Efimov, Opt. Express 18, 25329 (2010).

${ }^{19}$ V. Yannopapas and A. Vanakaras, Phys. Rev. B 84, 045128 (2011).

${ }^{20}$ D. Han, Y. Lai, J. Zi, Z. Q. Zhang, and C. T. Chan, Phys. Rev. Lett. 102, 123904 (2009).

${ }^{21}$ P. M. Hui, W. M. Lee, and N. F. Johnson, Solid State Commun. 91, 65 (1994).

${ }^{22}$ J. Mathews and R. L. Walker, Mathematical Methods of Physics, 2nd ed. (Addison-Wesley, Reading, MA, 1970).

${ }^{23}$ B. A. Foreman, J. Phys.: Condens. Matter 12, R435 (2000).

${ }^{24}$ M. Kafesaki and E. N. Economou, Phys. Rev. B 60, 11993 (1999).

${ }^{25}$ D. Torrent and J. Sanchez-Dehesa, New J. Phys. 13, 093018 (2011).

${ }^{26}$ Y. Wu and Z. Q. Zhang, Phys. Rev. B 79, 195111 (2009).

${ }^{27}$ M. S. Dresselhaus, G. Dresselhaus, and A. Jorio, Group Theory: Application to the Physics of Condensed Matter (Springer-Verlag, Berlin, Heidelberg, 2008).

${ }^{28}$ J. J. Sakurai, Modern Quantum Mechanics (Addison-Wesley, Reading, MA, 1994). 